\title{
Prevalencia y manejo de anemia en enfermedad inflamatoria intestinal en un centro de referencia en Colombia
}

\section{Prevalence and treatment of anemia in inflammatory bowel disease in a Colombian reference center}

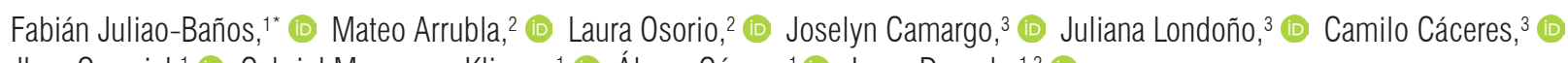
Jhon Carvajal, ${ }^{1}$ (1) Gabriel Mosquera-Klinger, ${ }^{1}$ [D Álvaro Gómez, ${ }^{1}$ (1) Jorge Donado., ${ }^{1,2}$ ()

\begin{abstract}
GACCESO ABIERTO
Citación:

Juliao-Baños F, Arrubla M, Osorio L, Camargo J, Londoño J, Cáceres C, Carvajal J, MosqueraKlinger G, Gómez A, Donado J. Prevalencia y manejo de anemia en enfermedad inflamatoria intestinal en un centro de referencia en Colombia. Rev Colomb Gastroenterol. 2021;36(4):446-454 https://doi.org/10.22516/25007440.696
\end{abstract}

Hospital Pablo Tobón Uribe. Medellín, Colombia. Universidad Pontificia Bolivariana. Medellin, Colombia.

${ }^{3}$ Universidad de Antioquia. Medellín, Colombia.

*Correspondencia: Fabián Juliao-Baños. fabianjuliao@hotmail.com

Fecha recibido: 22/11/20 Fecha aceptado: $12 / 02 / 21$

\section{Resumen}

Introducción: la anemia es la complicación más frecuente en enfermedad inflamatoria intestinal (EII). El objetivo de este estudio fue determinar la prevalencia, asociaciones y tratamiento de la anemia en Ell en nuestro medio. Materiales y métodos: se analizaron de forma retrospectiva datos de pacientes adultos con Ell del Hospital Pablo Tobón Uribe en Medellín, Colombia, que consultaron desde 2001 hasta febrero de 2019. Se emplearon frecuencias absolutas y relativas. Para comparar dos proporciones se utilizó la prueba de chi cuadrado de independencia y se estimó el Odds ratio (OR). Resultados: se documentaron 759 pacientes con Ell, de los cuales 544 $(71,6 \%)$ presentaron colitis ulcerativa (CU); 200 (26,3\%), enfermedad de Crohn (EC), y 15, Ell no clasificable $(1,9 \%)$. En total, $185(24,4 \%)$ pacientes con Ell presentaron anemia, que es menos frecuente en CU que en EC (22,2 \% frente a 32,5\%, respectivamente; OR: 0,684; IC: 0,456-0,96; $p=0,03)$. Los pacientes con CU extensa (54,1\%) tenían más anemia que aquellos con CU no extensa (46,3\%) (OR: 4,4; IC: 2,6-7,4; $p=0,001$ ); lo mismo se observó al comparar la CU grave (66,1\%) con la no grave (32,3\%) (OR: 4,95; IC: $2,87-8,51 ; p=0,000)$. En la $\mathrm{EC}$, los pacientes con comportamiento no inflamatorio (B2, B3: 73,9\%) presentaron más anemia en comparación con aquellos con comportamiento inflamatorio (B1: 26,2 \%) (OR: 0,35; IC: 0,18-0,67; $p=0,000)$. 44,3\% de los pacientes no recibió tratamiento, $19,5 \%$ recibió hierro oral, $20,0 \%$ recibió hierro intravenoso y $16,2 \%$ fue transfundido. Conclusiones: la anemia es una complicación frecuente de la Ell en nuestro medio $(24,4 \%)$. A pesar de las guías internacionales, el tratamiento es subóptimo en nuestro medio.

\section{Palabras clave}

Anemia, enfermedad inflamatoria intestinal, prevalencia, hierro parenteral.

\begin{abstract}
Introduction: Anemia is the most frequent complication of inflammatory bowel disease (IBD). This study aims to determine the prevalence, connection, and treatment of anemia in IBD in a local context. Materials and Methods: This retrospective study was conducted at The Pablo Tobon Uribe Hospital, in Medellín (Colombia) with adult patients who (were admitted) came for consultation from 2001, until February 2019. Absolut and relative frequencies were calculated. The Chi square test of independence was applied for comparing two proportions and the odds ratio (OR) was estimated. Results: A total of 759 IBD patients were enrolled, $544(71.6 \%)$ with ulcerative colitis (UC); 200 (26.3\%) Crohn's disease (CD), and 15 (1.9\%) with non-classifiable IBD. In total, 185 $(24,4 \%)$ IBD patients had a diagnosis of anemia, that is less frequent in UC patients that in CD patients $(22,2 \%$ versus $32,5 \%$, respectively; OR: 0,684; Cl: 0,456-0,96; $p=0,03)$. Extensive UC patients $(54,1 \%)$ had a more recurrent level of anemia than non-extensive UC (46,3\%) (OR: 4,4; Cl: 2,6-7,4; $p=0,001)$; the same result was observed when severe UC (66,1\%) was compared with UC non-severe (32,3\%) (OR: 4,95; Cl: 2,87-8,51; $p=$ 0,000 ). In the analysis of $C D$, patients with a non-inflammatory response (B2, B3: 73,9\%) had a more recurrent level of anemia than patients with an inflammatory response (B1: $26,2 \%)(\mathrm{OR}: 0,35 ; \mathrm{Cl}: 0,18-0,67 ; p=0,000)$. $44,3 \%$ of the total number of patients received treatment, $19,5 \%$ received oral iron, $20,0 \%$ received intravenous iron, and $16,2 \%$ received a blood transfusion. Conclusions: In our context, Anemia is a common complication in IBD cases $(24,4 \%)$. Despite the existence of international guidelines, the treatment in our context is not optimal.
\end{abstract}

\section{Keywords}

Anemia; Inflammatory Bowel Disease; Prevalence; Parenteral Iron. 


\section{INTRODUCCIÓN}

La anemia es la complicación más frecuente de la enfermedad inflamatoria intestinal (EII) ${ }^{(1)}$, se asocia con una enfermedad más discapacitante ${ }^{(2)}$ con un impacto significativo en la calidad de vida ${ }^{(3,4)}$ e históricamente ha recibido poca atención por parte de los gastroenterólogos ${ }^{(5)}$. La anemia se define por la Organización Mundial de la Salud (OMS) como los niveles de hemoglobina $(\mathrm{Hb})<12 \mathrm{~g} / \mathrm{dL}$ en mujeres no embarazadas $\mathrm{y}<13 \mathrm{~g} / \mathrm{dL}$ en hombres ${ }^{(6)}$. La anterior definición de anemia fue adoptada por la Organización Europea de Enfermedad de Crohn y Colitis ulcerativa (ECCO) en un consenso reciente, en el que se recomienda descartar esta complicación en todos los individuos con $\mathrm{EII}^{(1)}$.

La etiología de la anemia en EII es multifactorial y la causa más común es la anemia por deficiencia de hierro $(\mathrm{ADH})$ y la anemia asociada a enfermedad crónica (AEC), y estas pueden coexistir (mixta); otras causas de anemia como las asociadas con la deficiencia de vitamina $\mathrm{B}_{12} \mathrm{o}$ ácido fólico, o inducidas por medicamentos son menos frecuentes ${ }^{(7,8)}$. En una revisión sistemática de 17 artículos con EC, la prevalencia oscila entre $4 \%$ y $67 \%{ }^{(9)}$. Gisbert y colaboradores reportaron $17 \%$ de prevalencia promedio de anemia en EII y se encontraron cifras de $16 \%$ en pacientes ambulatorios y $68 \%$ en individuos hospitalizados $^{(10)}$. La prevalencia de EII en Latinoamérica está aumentado $^{(11)}$ y existen pocos datos de la relación entre la anemia y la EII. Dos estudios en Brasil encontraron una prevalencia de anemia de $21 \%$ y $24,6 \%$ en pacientes con $\mathrm{EII}^{(12,13)}$. El objetivo de este estudio es determinar la prevalencia, asociaciones y tratamiento de la anemia en una cohorte de pacientes con EII en nuestro centro.

\section{MATERIALES Y MÉTODOS}

Se buscaron los diagnósticos de CU y EC en las historias clínicas del Hospital Pablo Tobón Uribe en Medellín, Colombia, hasta febrero de 2019 teniendo en cuenta los siguientes códigos: K500 EC de intestino delgado, K501 EC del intestino grueso, K508 otros tipos de enfermedad de Crohn, K509 EC no especificada, K519 CU sin otra especificación y K518 otras CU. Se analizaron de forma retrospectiva los datos de pacientes adultos con CU y EC que asistieron por urgencias, hospitalizados o por consulta externa para determinar la presencia de anemia.

\section{Definiciones de anemia}

Se diagnosticó anemia en aquellos pacientes con niveles de $\mathrm{Hb}<12 \mathrm{~g} / \mathrm{dL}$ en mujeres no embarazadas $\mathrm{y}<13 \mathrm{~g} / \mathrm{dL}$ en hombres, según la recomendación de la $\mathrm{OMS}^{(6)}$. Se consi- deró $\mathrm{ADH}$ a los niveles de ferritina $<30 \mathrm{mg} / \mathrm{L}$ y niveles de proteína C-reactiva (PCR) normales, o en caso de ferritina $<100 \mathrm{mg} / \mathrm{L}$ y PCR elevada, pero con un porcentaje de saturación de transferrina $<16 \%$. La AEC se definió con niveles de ferritina normal o elevados, porcentaje de saturación de transferrina normal y PCR elevada ${ }^{(8)}$. La anemia por deficiencias de vitamina $\mathrm{B}_{12}(<211 \mathrm{ng} / \mathrm{mL})$ y ácido fólico $(<7 \mathrm{ng} / \mathrm{mL})$ se definieron cuando los niveles se encontraban por debajo del nivel normal. Otras mediciones como el volumen corpuscular medio (VCM), el ancho de distribución de glóbulos rojos (RDW) y el porcentaje de reticulocitos también se tuvieron en cuenta para clasificar la anemia ${ }^{(14,15)}$. Los pacientes en los que no se pudo definir la presencia de anemia por no tener datos suficientes se excluyeron del estudio.

Se consideró anemia leve a los niveles de $\mathrm{Hb}$ entre 11,0 y $11,9 \mathrm{~g} / \mathrm{dL}$ en mujeres y entre 11,0 y 12,9 en hombres, moderada entre 8,0 y $10,9 \mathrm{~g} / \mathrm{dL}$ y grave con niveles $<8 \mathrm{~g} /$ $\mathrm{dL}$, según las recomendaciones de la $\mathrm{OMS}^{(1,6)}$. La actividad de la CU se definió por la clasificación de Truelove y Witts ${ }^{(16)}$ y para el análisis se consideró la actividad presente al momento de la mayor gravedad de anemia encontrada. La extensión de CU se definió según la Clasificación de Montreal ${ }^{(17)}$. La localización y el comportamiento de EC se determinaron según la Clasificación de Montreal ${ }^{(17)}$.

\section{Recolección de datos}

Se construyó una base de datos con formato Excel, se recolectaron los siguientes datos de cada paciente para el análisis:

- Tipo de EII (CU, EC y EII no clasificable).

- Sexo del paciente.

- Extensión anatómica de la CU.

- Actividad de la CU.

- Localización de la EC.

- Comportamiento de la EC.

- Tratamiento médico acumulado (ácido 5 aminosalicílico [5-ASA], esteroides, inmunosupresores, terapia biológica).

- Tratamiento quirúrgico.

- Tasa de hospitalización.

- Presencia de anemia.

- Tipo de anemia.

- Gravedad de la anemia.

- Tratamiento de la anemia.

Para clasificar la anemia se tuvieron en cuenta las siguientes mediciones: $\mathrm{Hb}$, hematocrito, VCM, ferritina sérica, porcentaje de saturación de transferrina y PCR. En los pacientes con elevación de VCM se verificaban los niveles de vitamina $\mathrm{B}_{12} \mathrm{y}$ ácido fólico. 


\section{Análisis estadístico}

Se emplearon frecuencias absolutas y relativas para las variables cualitativas, y para las variables cuantitativas se utilizaron media y desviación estándar (DE) o mediana y rango intercuartílico (RIC; P25-P75) previa verificación del supuesto de normalidad con las pruebas KolmogorovSmirnov. Para comparar dos proporciones se utilizó la prueba de chi cuadrado $\left(\chi^{2}\right)$ de independencia y se estimó el Odds ratio (OR) con su respectivo intervalo de confianza (IC) del $95 \%$.

\section{Consideraciones éticas}

Esta es una investigación sin riesgo, ya que se revisaron las historias clínicas de los pacientes y se garantizó la confidencialidad y privacidad de la información recogida. Los investigadores del proyecto se adhirieron a los principios internacionales de la Declaración de Helsinki versión 2013 en Fortaleza, Brasil, y con los artículos 10 y 11 de la resolución 008430 de 1993 del Ministerio de Salud Nacional de Colombia.

\section{RESULTADOS}

Este es un estudio retrospectivo, descriptivo y analítico, en el que se incluyeron sistemáticamente 759 pacientes que cumplían criterios diagnósticos de EII, de los cuales 544 (71,6\%) presentaron diagnóstico de CU, 200 (26,3\%) de EC y $15(1,9 \%)$ de EII no clasificable.

Se documentó anemia en 185 delos 759 pacientes $(24,4 \%)$ con EII. En CU, 121 de 544 (22,2 \%) presentaron anemia, comparado con 65 de 200 pacientes $(32,5 \%)$ en EC (OR: $0,684$; IC: $0,456-0,96 p=0,03)$. En cuanto a anemia grave, se presentó en 87 de 185 pacientes $(47,0 \%)$ con EII, 61 de $121(50,4 \%)$ con CU y 26 de $65(40,0 \%)$ con EC. Del total de pacientes con anemia, 102 tenían $\mathrm{ADH}(55,1 \%)$, $74(41,1 \%)$ tenían anemia mixta y $7(3,7 \%)$ presentaron déficit de vitamina $B_{12}$ (Figura 1).

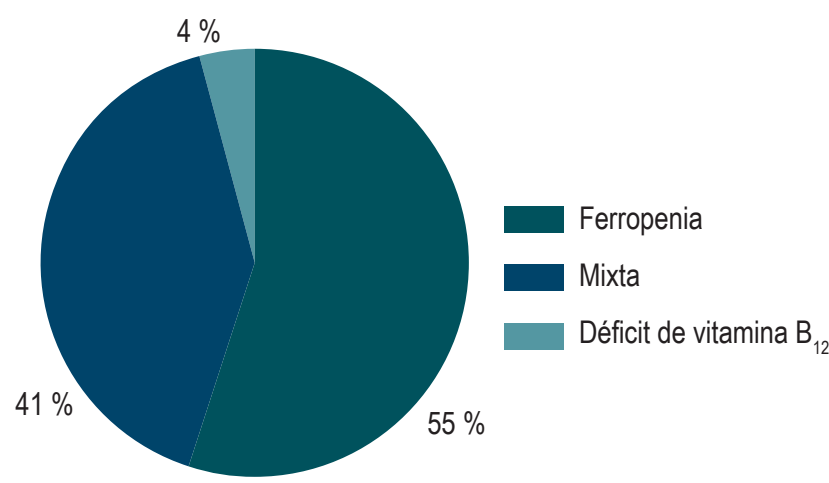

Figura 1. Tipo de anemia en EII.
De los pacientes con CU y anemia, 18,2\% presentaron proctitis, $28,1 \%$ colitis izquierda y $54,1 \%$ colitis extensa. En la comparación de la anemia en colitis extensa frente a la no extensa (proctitis más colitis izquierda), se encontró una diferencia significativa (OR: 4,4; IC: 2,6-7,4 $p=$ $0,001)$. En cuanto a la actividad de CU, el 5,0 \% se encontraba asintomático (S0), 14,0 \% con actividad leve (S1), $15,7 \%$ con actividad moderada ( 22 ) y $66,1 \%$ con actividad grave (S3). Al comparar la presencia de anemia en actividad grave frente a la no grave, se encontró una diferencia significativa (OR: 4,95 ; IC: $2,87-8,51 ; p=0,000$ ).

Teniendo en cuenta la localización, la distribución de anemia en pacientes con EC fue la siguiente: ileal (40\%), ileocolónica $(35,4 \%)$, colónica $(20 \%)$ y con compromiso digestivo superior $(4,6 \%)$. No se encontró una diferencia significativa entre la localización colónica frente a la no colónica (OR: 1,2; IC: 0,66-2,19; $p=0,54$ ). En cuanto al comportamiento de la EC y la presencia de anemia, en el $26,2 \%$ es inflamatorio (B1), el 27,7 \% estenosante (B2), el $27,7 \%$ penetrante (B3) y $18,5 \%$ perianal. El comportamiento inflamatorio presentó menos frecuentemente anemia, comparado con el comportamiento no inflamatorio (B2 más B3) (OR: 0,35; IC: 0,18-0,67; $p=0,000)$.

Los pacientes con $\mathrm{CU}$ y anemia requirieron más terapia biológica que aquellos sin anemia $(33,1 \%$ frente a $14,4 \%)$; esta diferencia fue significativa (OR: 2,29 ; IC: $1,46-3,58 ; p=$ $0,009)$. En $E C$, los pacientes con anemia también requirieron más terapia biológica (61,5\% frente a 39,3\%); sin embargo, esta diferencia no fue significativa (OR: 1,56; IC: 0,94-2,6; $p=0,08)$. No hubo diferencia significativa en cuanto a la necesidad de cirugía en pacientes con anemia frente a no anemia en CU (11,5\% frente a 11,1\%; OR: 1,04 ; IC: $0,55-$ $1,95 ; p=0,87)$, ni en EC $(46,2 \%$ frente a $34,8 \%$; OR: 1,32 ; IC: $0,76-2,28 ; p=0,32$ ). En cuanto a la hospitalización, los pacientes con $\mathrm{CU}$ y anemia requirieron más hospitalización en comparación con aquellos sin anemia $(59,5 \%$ frente a $47,0 \%$; OR: 1,95 ; IC: $1,37-2,77 ; p=0,000)$. Lo anterior no se demostró en individuos con EC (72,3\% frente a $63,0 \%$, OR: 1,14; IC: 0,72-1,82; $p=0,63$ ) (Tabla 1).

En cuanto al tratamiento de anemia y EII, 82 de 185 $(44,3 \%)$ pacientes con anemia no recibieron tratamiento, $19,5 \%$ recibieron hierro oral, $20,0 \%$ hierro intravenoso (IV) y 16,2\% tuvo transfusión (Figura 2). En el subgrupo de pacientes con anemia grave, 24 de $87(27,6 \%)$ no se trataron, $12,6 \%$ recibió hierro oral, $29,9, \%$ recibió hierro IV y $29,9 \%$ tuvo transfusión. Ningún paciente recibió eritropoyetina.

\section{DISCUSIÓN}

La prevalencia de anemia en 759 pacientes adultos con EII, tanto ambulatorios como hospitalizados, en nuestro 
Tabla 1. Características clínicas de EII y anemia

EC

Pacientes (n)
200
CU

$p$

544

$\begin{array}{cccc}\text { Anemia } & \text { No anemia } & \text { Anemia } & \text { No anemia } \\ 65(32,5 \%) & 135(67,5 \%) & 121(22,2 \%) & 423(77,8 \%)\end{array}$

0,03

Extensión de la CU

- E1: proctitis + E2: izquierda

$56(45,9 \%)$

0,001

$65(54,1 \%)$

- E3: colitis extensa

$26(40,0 \%)$

- L1: íleon terminal

$23(35,4 \%)$

- L3: íleon terminal + colon

$3(4,6 \%)$

- L4: GI superior

$13(20,0 \%)$

- L2: Colon

$40(33,9 \%)$

0,000

- Actividad no grave (S1 + S2)

$81(66,1 \%)$

- Actividad grave (S3)

Comportamiento de la EC

- B1: inflamatoria

$17(26,2 \%)$

0,000

- $B 2+B 3+P$

$48(73,9 \%)$

Biológico/cirugía/hospitalización por CU

- Biológico

$(73,9 \%)$

- Cirugía

- Hospitalización

Biológico/cirugia/hospitalización por EC

- Biológico

- Cirugía

- Hospitalización

Anemia
$40(61,5 \%)$
$30(46,2 \%)$
$47(72,3 \%)$
Anemia

$40(33,1 \%)$

$14(11,5 \%)$

$72(59,5 \%)$

No anemia

$53(39,3 \%)$

$47(34,8 \%)$

$85(63 \%)$
0,08

0,32

0,009

0,87

0,000

0,63 centro es alta (24,4\%), y es más frecuente en EC (32,5\%) que en CU $(22,2 \%)$, datos similares a lo reportado en la literatura universal. Un metaanálisis europeo con 2192 pacientes encontró una prevalencia de anemia en el $24 \%$ de casos con EII, $27 \%$ de casos con EC y $21 \%$ de casos con $\mathrm{CU}^{(18)}$. El grupo noruego IBSEN encontró también una mayor proporción de anemia al diagnóstico en $\operatorname{EC~(48,8,~\% ),~}$ en comparación con CU $(20,2 \%)^{(19)}$. Un estudio sueco encontró $30 \%$ de anemia en pacientes con EII al diagnóstico, $42 \%$ en pacientes con EC y $24 \%$ en pacientes con
$\mathrm{CU}^{(20)}$. Una publicación más reciente de la Universidad de Pittsburgh encontró una prevalencia de anemia en pacientes con EII de 33,2 \%, 34,3 \% en pacientes con EC y $31,5 \%$ en pacientes con $\mathrm{CU}^{(2)}$. El estudio de cohorte ECCOEPICOM encontró una prevalencia mayor de anemia en pacientes con EC de $49 \%$ y $39 \%$ en pacientes con CU durante el primer año del diagnóstico ${ }^{(21)}$.

Llamó la atención la alta cantidad de pacientes con anemia grave en nuestros pacientes con EII (40,0 \%), en comparación con otras series. En un estudio multicéntrico en 


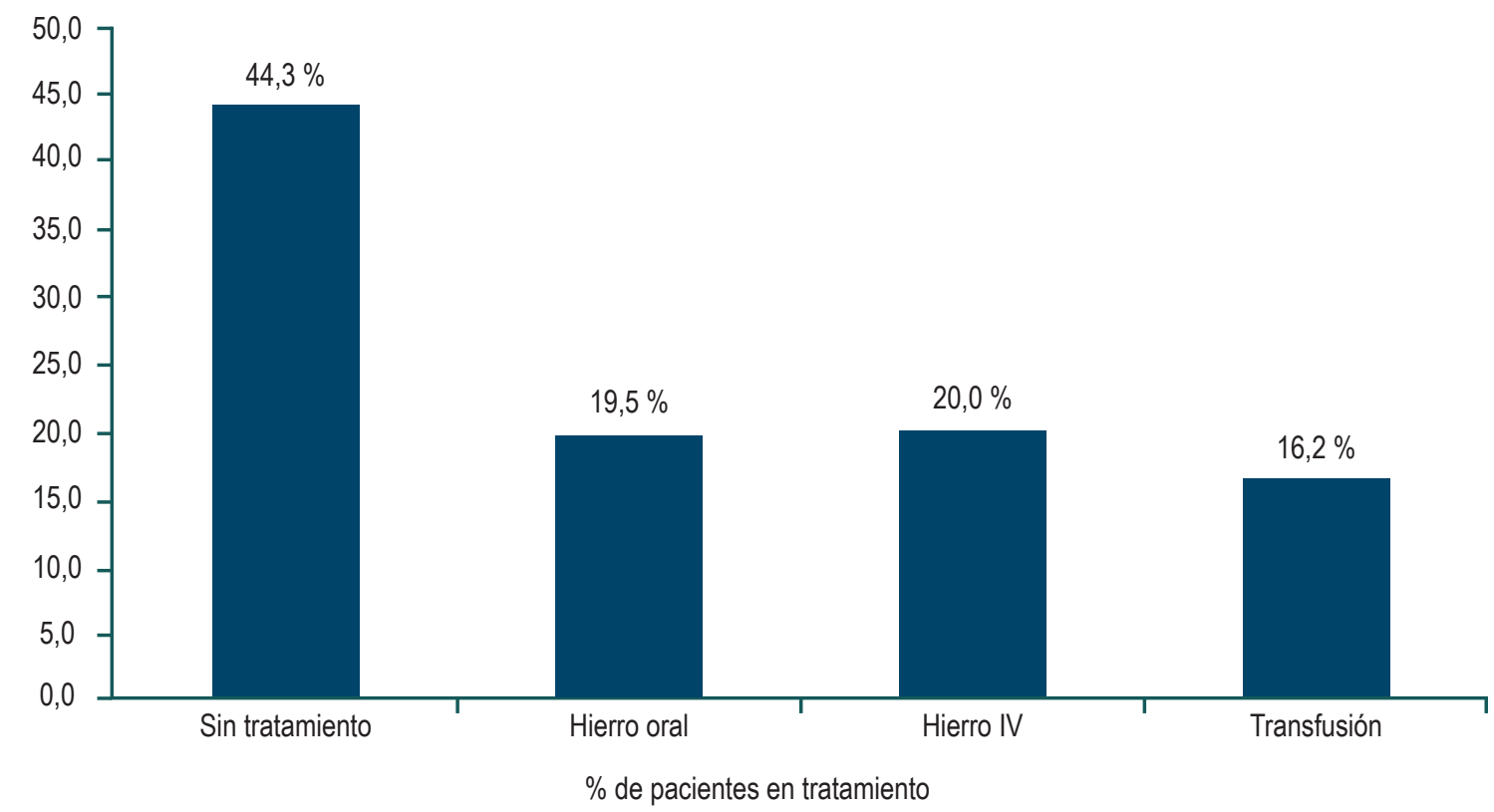

Figura 2. Tratamiento de la anemia y EII.

9 países europeos con 1404 pacientes con EII se observó la presencia del $56 \%$ de individuos con al menos anemia moderada $(\mathrm{Hb}<10 \mathrm{~g} / \mathrm{dL})$, pero solo el $15 \%$ tuvo anemia grave $^{(22)}$. La gravedad de la anemia fue leve en el $76,2 \%$, moderada en el $15 \%$ y grave en el $8,8 \%$, en un registro de 193 casos de anemia asociada con EII, en 55 centros de Alemania ${ }^{(23)}$. Lo anterior puede explicarse por la gravedad de los pacientes con EII que se atienden en nuestro centro de alta complejidad; estas dos series son encuestas que se hicieron a los participantes y no se realizó análisis de la gravedad de EII en sus pacientes.

Por una parte, nuestro estudio demostró un predominio de $\mathrm{ADH}(55 \%)$ y mixta (41\%) sobre otras etiologías de anemia. En un estudio escandinavo ${ }^{(24)}$, la etiología de anemia en EII fue ADH (20\%), AEC (12\%), mixta (68\%) y déficit de vitamina $B_{12} y$ ácido fólico $(<5 \%)$, similar a lo encontrado en nuestro estudio, pero con una mayor proporción de anemia mixta.

Por otra parte, en nuestro estudio los pacientes con CU y anemia presentaban actividad más grave (S3), mayor extensión anatómica y enfermedad más discapacitante, debido a una mayor tasa de hospitalización y mayor uso de terapia biológica. En individuos con EC, la presencia de anemia se asoció con el comportamiento no inflamatorio (estenosante o penetrante), por lo que estos pacientes son más complicados y de peor pronóstico en comparación con pacientes con comportamiento inflamatorio. En el estudio ECCO-EPICOM, ya señalado, se encontró que los pacien- tes con CU extensa y EC penetrante presentaron más riesgo de anemia, similar a lo demostrado en este estudio ${ }^{(21)}$. En el estudio sueco mencionado previamente, la presencia de anemia se asoció también con CU extensa ${ }^{(20)}$. Datos del estudio de la Universidad de Pittsburgh ya señalado muestran también una correlación significativa de anemia con índices más altos de actividad de la enfermedad y de hospitalización ${ }^{(2)}$.

Las guías de $\mathrm{ECCO}^{(1)}$ recomiendan el uso de hierro oral en pacientes con EII con ADH leve definida por la OMS con niveles de $\mathrm{Hb}$ de 11-11-9 g/dL, cuya EII se encuentre clínicamente inactiva y con buena tolerancia al medicamento. El hierro IV debe ser considerado como primera línea de tratamiento en pacientes con intolerancia al hierro oral, EII activa, niveles de $\mathrm{Hb}<10 \mathrm{~g} / \mathrm{dL}$ y en pacientes que requieran eritropoyetina ${ }^{(1)}$. Un reciente artículo de revisión, teniendo en cuenta la pandemia por la enfermedad por coronavirus de 2019 (COVID-19), sugiere utilizar durante estos tiempos hierro oral en pacientes con EII y anemia leve y moderada, para evitar que los pacientes con EII asistan a centros hospitalarios para colocarse hierro parenteral, y reservar este último solo para sujetos con EII y anemia grave $(\mathrm{Hb}<8 \mathrm{~g} / \mathrm{dL})^{(25)}$.

En cuanto al tratamiento de la $\mathrm{ADH}$ en este estudio, el $44,3 \%$ de los pacientes no recibió tratamiento y un alto porcentaje del subgrupo de individuos con anemia grave tampoco $(27,6 \%)$. Consideramos que aún se maneja el concepto de anemia asintomática en el cuerpo médico, sin 
tener en cuenta la alteración en la calidad de vida a la que conlleva esta complicación. En una encuesta previa a gastroenterólogos de toda Colombia, ante la pregunta de cuál sería el mejor manejo de un paciente con EII y $\mathrm{ADH}<10$ $\mathrm{g} / \mathrm{dL}$, la respuesta fue que el $66 \%$ la trataría con hierro oral, $15 \%$ con hierro parenteral, $9 \%$ transfunde hierro y $10 \%$ no la trata ${ }^{(26)}$. En el estudio multicéntrico alemán mencionado anteriormente, solo el 43,5\% de pacientes con ADH yEII ha recibido tratamiento; de ellos, el $56 \%$ con hierro oral, $15 \%$ con hierro parenteral y $10 \%$ en transfusión ${ }^{(23)}$. En Reino Unido se realizó una encuesta a pacientes ambulatorios con anemia y EII que recibieron hierro oral, solo el $42 \%$ completó el tratamiento, y en 2 de 3 pacientes el tratamiento no fue efectivo para el control de la anemia ${ }^{(27)}$. En el estudio europeo mencionado previamente, $92 \%$ de los pacientes con $\mathrm{ADH}$ recibió suplemento de hierro, $67 \%$ hierro oral y solo $28 \%$ hierro IV, a pesar de que el $56 \%$ presentaba $\mathrm{Hb}<$ $10 \mathrm{~g} / \mathrm{dL}^{(21)}$. Un estudio retrospectivo realizado en Estados Unidos encontró que, en pacientes anémicos con EII, solo el $37 \%$ recibió hierro oral y 2,8 \% hierro IV durante el seguimiento ${ }^{(28)}$. Una encuesta mundial a pacientes con anemia y EII encontró que el 33 \% refirió que no recibió tratamiento para su anemia, y de los tratados, el $52 \%$ recibió hierro oral, $27 \%$ hierro IV y $19 \%$ otro tipo de suplementos. De los pacientes con hierro oral, el $74 \%$ no estaba satisfecho con el tratamiento; mientras que el $72 \%$ de los pacientes con el hierro IV estaba satisfecho ${ }^{(29)}$.

Recientemente, la Fundación Estadounidense para la Enfermedad de Crohn y la Colitis Ulcerativa (CCFA) asignó un comité de expertos, quienes elaboraron recomendaciones y un algoritmo de tamizaje, evaluación, intervención y seguimiento de pacientes con anemia y EII ${ }^{(30)}$. Una publicación posterior evaluó la adherencia a estas recomendaciones y encontró que la terapia con hierro en pacientes anémicos se incrementó del $30 \%$ al $80 \%$ y la prevalencia de anemia disminuyó del 48 \% al $25 \%$; sin embargo, el porcentaje de tamizaje de deficiencia de hierro no cambió: a solo el $20 \%$ de los pacientes anémicos se les realizó una medición de ferritina ${ }^{(31)}$. Un artículo de revisión reciente recomienda tratar hacia un objetivo a pacientes con $\mathrm{ADH}$ asociado a EII (treat to target), similar a lo propuesto para el tratamiento de la EII, dividido en tres pasos: detección temprana, tratar hacia un objetivo (normalización de $\mathrm{Hb}$ y depósitos de hierro) y monitorización estricta (ferritina y porcentaje de saturación de transferrina cada 3-6 meses) ante el alto riesgo de recurrencia ${ }^{(32)}$.

Dentro de las limitaciones de este estudio se puede decir que, al ser un estudio retrospectivo y basado en datos recolectados de la historia clínica, puede contener sesgos de interpretación. Adicionalmente, este estudio fue realizado en un hospital de alta complejidad, centro de referencia de pacientes con EII de todo el país, y probablemente se incluyeron pacientes más graves y complicados en comparación con pacientes de otros centros del país.

\section{CONCLUSIONES}

Existe una alta prevalencia de anemia asociada con EII en nuestro medio, la cual es más frecuente en EC que en CU, lo que es consistente con resultados de otros estudios a nivel mundial. Lo anterior se podría explicar por un diagnóstico insuficiente, un tratamiento inefectivo y una falta de monitorización en estos individuos con anemia. La presencia de anemia en pacientes con EII se asocia con una mayor gravedad de la enfermedad. Adicionalmente, la ruta de administración preferida de hierro en $\mathrm{ADH}$, tanto en esta como en otras series citadas, es la vía oral, independiente de la gravedad de la anemia, y a pesar del alto porcentaje de intolerancia al hierro oral y de las recomendaciones de guías internacionales. Definitivamente, es necesario tomar más conciencia de esta complicación en los pacientes con EII y realizar más esfuerzos en educación médica, para implementar recomendaciones de guías y aplicarlas en el diagnóstico temprano, manejo adecuado y monitorización de estos individuos. Sin embargo, lo más importante va a ser siempre el tratamiento oportuno y adecuado de la EII para prevenir esta complicación. Una propuesta para el manejo de anemia en pacientes con EII se presenta en la Figura 3.

\section{Conflictos de interés}

Ninguno de los autores del estudio reportó conflictos de interés.

\section{Fuente de financiación}

Para la realización de este estudio, no se recibió financiación de ninguna entidad.

\section{Contribución de autores}

F. Juliao-Baños F participó en el diseño del estudio, reclutamiento de pacientes y redacción del documento. $\mathrm{M}$. Arrubla L. Osorio, J. Camargo J. Londoño y C. Cáceres realizaron la recolección de datos. J. Carvajal, G. Mosquera y A. Gómez participaron en el reclutamiento de pacientes. J. Donado realizó el análisis estadístico. 


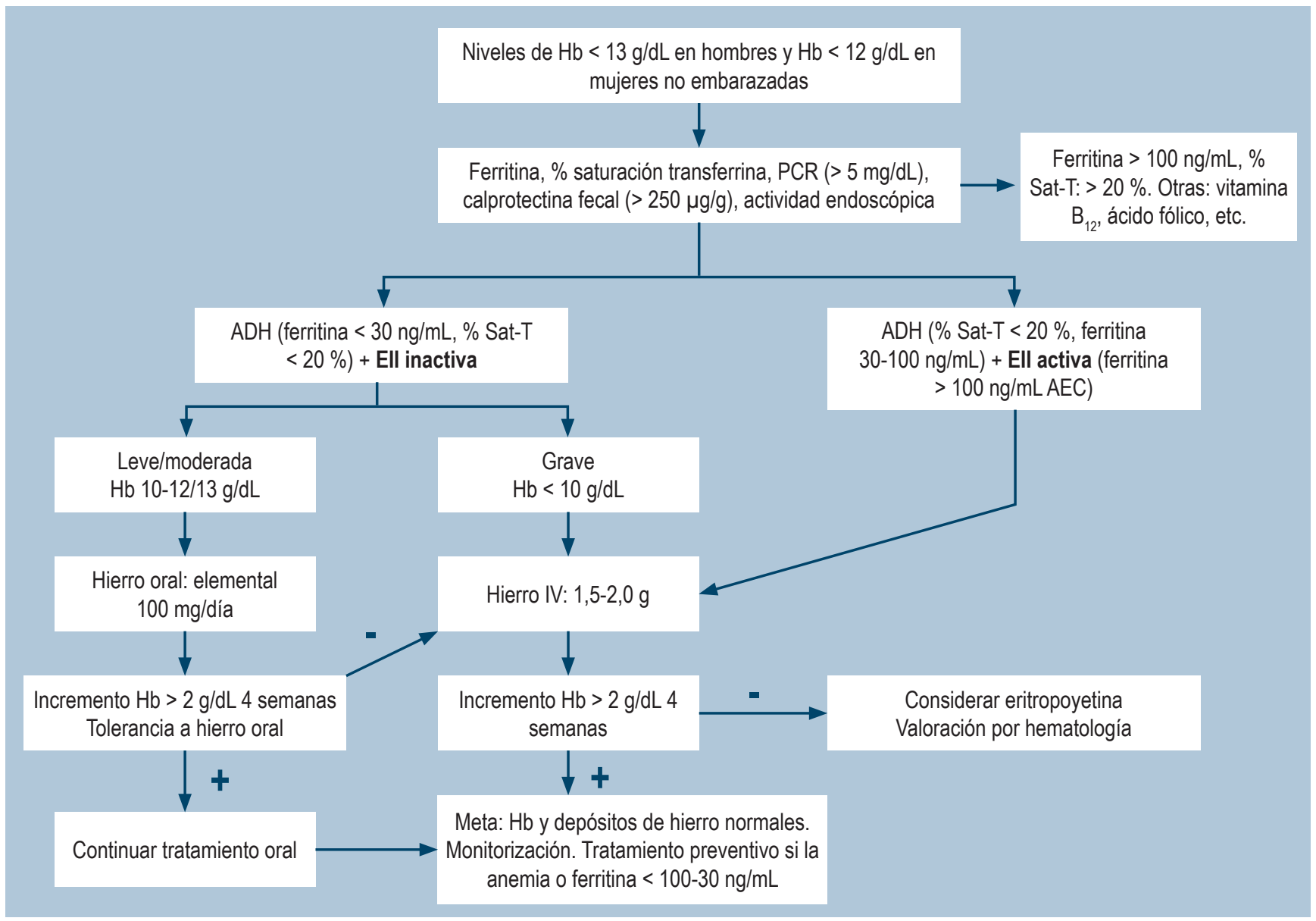

Figura 3. Algoritmo manejo anemia en EII. Sat-T: saturación de transferrina.

\section{REFERENCIAS}

1. Dignass AU, Gasche C, Bettenworth D, Birgegård G, Danese S, Gisbert JP, Gomollon F, Iqbal T, Katsanos K, Koutroubakis I, Magro F, Savoye G, Stein J, Vavricka S; European Crohn's and Colitis Organisation [ECCO]. European consensus on the diagnosis and management of iron deficiency and anaemia in inflammatory bowel diseases. J Crohns Colitis. 2015;9(3):211-22.

https://doi.org/10.1093/ecco-jcc/jju009

2. Koutroubakis IE, Ramos-Rivers C, Regueiro M, Koutroumpakis E, Click B, Schoen RE, Hashash JG, Schwartz M, Swoger J, Baidoo L, Barrie A, Dunn MA, Binion DG. Persistent or Recurrent Anemia Is Associated With Severe and Disabling Inflammatory Bowel Disease. Clin Gastroenterol Hepatol. 2015;13(10):1760-6. https://doi.org/10.1016/j.cgh.2015.03.029

3. Wells CW, Lewis S, Barton JR, Corbett S. Effects of changes in hemoglobin level on quality of life and cognitive function in inflammatory bowel disease patients. Inflamm
Bowel Dis. 2006; 12(2):123-30. https://doi.org/10.1097/01.MIB.0000196646.64615.db

4. González Alayón C, Pedrajas Crespo C, Marín Pedrosa S, Benítez JM, Iglesias Flores E, Salgueiro Rodríguez I, Medina Medina R, García-Sánchez V. Prevalence of iron deficiency without anaemia in inflammatory bowel disease and impact on health-related quality of life. Gastroenterol Hepatol. 2018;41(1):22-29. https://doi.org/10.1016/j.gastrohep.2017.07.011

5. Gasche C. Anemia in IBD: the overlooked villain. Inflamm Bowel Dis. 2000;6(2):142-150. https://doi.org/10.1097/00054725-200005000-00013

6. WHO U, UNU. Iron Deficiency Anemia: Assessment, Prevention and Control. Report of a Joint WHO/ UNICEF/UNU Consultation. Geneva, Switzerland: World Health Organization 2001. Disponible en: https://www. who.int/nutrition/publications/en/ida_assessment_prevention_control.pdf 
7. Stein J, Hartmann F, Dignass AU. Diagnosis and management of iron deficiency anemia in patients with IBD. Nat Rev Gastroenterol Hepatol. 2010;7(11):599-610. https://doi.org/10.1038/nrgastro.2010.151

8. Oustamanolakis P, Koutroubakis IE, Kouroumalis EA. Diagnosing anemia in inflammatory bowel disease: beyond the established markers. J Crohns Colitis. 2011;5(5):381-91. https://doi.org/10.1016/j.crohns.2011.03.010

9. Kulnigg S, Gasche C. Systematic review: managing anaemia in Crohn's disease. Aliment Pharmacol Ther. 2006;24(1112): 1507-23. https://doi.org/10.1111/j.1365-2036.2006.03146.x

10. Gisbert JP, Gomollón F. Common misconceptions in the diagnosis and management of anemia in inflammatory bowel disease. Am J Gastroenterol. 2008;103(5):1299-307. https://doi.org/10.1111/j.1572-0241.2008.01846.x

11. Kotze PG, Underwood FE, Damião AOMC, Ferraz JGP, Saad-Hossne R, Toro M, Iade B, Bosques-Padilla F, Teixeira FV, Juliao-Banos F, Simian D, Ghosh S, Panaccione R, $\mathrm{Ng}$ SC, Kaplan GG. Progression of Inflammatory Bowel Diseases Throughout Latin America and the Caribbean: A Systematic Review. Clin Gastroenterol Hepatol. 2020;18(2):304-312. https://doi.org/10.1016/j.cgh.2019.06.030

12. Antunes CV, Hallack Neto AE, Nascimento CR, Chebli LA, Moutinho IL, Pinheiro Bdo V, Reboredo MM, Malaguti C, Castro AC, Chebli JM. Anemia in inflammatory bowel disease outpatients: prevalence, risk factors, and etiology. Biomed Res Int. 2015;2015:728925. https://doi.org/10.1155/2015/728925

13. Parra RS, Feitosa MR, Ferreira SDC, Rocha JJRD, Troncon LEA, FÉres O. Anemia and iron deficiency in inflammatory bowel disease patients in a referral center in brazil: prevalence and risk factors. Arq Gastroenterol. 2020;57(3):272-277. https://doi.org/10.1590/S0004-2803.202000000-51

14. Gasche C, Berstad A, Befrits R, Beglinger C, Dignass A, Erichsen K, Gomollon F, Hjortswang H, Koutroubakis I, Kulnigg S, Oldenburg B, Rampton D, Schroeder O, Stein J, Travis S, Van Assche G. Guidelines on the diagnosis and management of iron deficiency and anemia in inflammatory bowel diseases. Inflamm Bowel Dis. 2007;13(12):1545-53. https://doi.org/10.1002/ibd.20285

15. Reinisch W, Staun M, Bhandari S, Muñoz M. State of the iron: how to diagnose and efficiently treat iron deficiency anemia in inflammatory bowel disease. J Crohns Colitis. 2013;7(6):429-40. https://doi.org/10.1016/j.crohns.2012.07.031

16. Truelove Sc, Witts LJ. Cortisone in ulcerative colitis; final report on a therapeutic trial. Br Med J. 1955;2(4947):1041-8. https://doi.org/10.1136/bmj.2.4947.1041

17. Silverberg MS, Satsangi J, Ahmad T, Arnott ID, Bernstein CN, Brant SR, Caprilli R, Colombel JF, Gasche C, Geboes K, Jewell DP, Karban A, Loftus EV Jr, Peña AS, Riddell RH, Sachar DB, Schreiber S, Steinhart AH, Targan SR, Vermeire $S$, Warren BF. Toward an integrated clinical, molecular and serological classification of inflammatory bowel disease: report of a Working Party of the 2005 Montreal World Congress of Gastroenterology. Can J Gastroenterol. 2005; 19 Suppl A:5A-36A. https://doi.org/10.1155/2005/269076

18. Filmann N, Rey J, Schneeweiss S, Ardizzone S, Bager P, Bergamaschi G, Koutroubakis I, Lindgren S, Morena Fde L, Moum B, Vavricka SR, Schröder O, Herrmann E, Blumenstein I. Prevalence of anemia in inflammatory bowel diseases in european countries: a systematic review and individual patient data meta-analysis. Inflamm Bowel Dis. 2014;20(5):936-45. https://doi.org/10.1097/01.MIB.0000442728.74340.fd

19. Høivik ML, Reinisch W, Cvancarova M, Moum B; IBSEN study group. Anaemia in inflammatory bowel disease: a population-based 10-year follow-up. Aliment Pharmacol Ther. 2014;39(1):69-76. https://doi.org/10.1111/apt.12541

20. Sjöberg D, Holmström T, Larsson M, Nielsen AL, Holmquist L, Rönnblom A. Anemia in a population-based IBD cohort (ICURE): still high prevalence after 1 year, especially among pediatric patients. Inflamm Bowel Dis. 2014;20(12):2266-70. https://doi.org/10.1097/MIB.0000000000000191

21. Burisch J, Vegh Z, Katsanos KH, Christodoulou DK, Lazar D, Goldis A, et al; EpiCom study group. Occurrence of Anaemia in the First Year of Inflammatory Bowel Disease in a European Population-based Inception Cohort-An ECCOEpiCom Study. J Crohns Colitis. 2017;11(10):1213-1222. https://doi.org/10.1093/ecco-jcc/jjx077

22. Stein J, Bager P, Befrits R, Gasche C, Gudehus M, Lerebours E, Magro F, Mearin F, Mitchell D, Oldenburg $B$, Danese S. Anaemia management in patients with inflammatory bowel disease: routine practice across nine European countries. Eur J Gastroenterol Hepatol. 2013;25(12):1456-63. https://doi.org/10.1097/MEG.0b013e328365ca7f

23. Blumenstein I, Dignass A, Vollmer S, Klemm W, WeberMangal S, Stein J. Current practice in the diagnosis and management of IBD-associated anaemia and iron deficiency in Germany: the German AnaemIBD Study. J Crohns Colitis. 2014;8(10):1308-14. https://doi.org/10.1016/j.crohns.2014.03.010

24. Bager P, Befrits R, Wikman O, Lindgren S, Moum B, Hjortswang H, Dahlerup JF. The prevalence of anemia and iron deficiency in IBD outpatients in Scandinavia. Scand J Gastroenterol. 2011;46(3):304-9. https://doi.org/10.3109/00365521.2010.533382

25. D’Amico F, Peyrin-Biroulet L, Danese S. Oral Iron for IBD Patients: Lessons Learned at Time of COVID-19 Pandemic. J Clin Med. 2020;9(5):1536. https://doi.org/10.3390/jcm9051536

26. Juliao F, Agudelo Y, Yepes C. Variación en el cuidado de pacientes con Enfermedad Inflamatoria Intestinal (EII): resultado de una encuesta. Rev Col Gastroenterol. 2014;29(1):11-18. 
27. Lugg S, Beal F, Nightingale P, Bhala N, Iqbal T. Iron treatment and inflammatory bowel disease: what happens in real practice? J Crohns Colitis. 2014;8(8):876-80. https://doi.org/10.1016/j.crohns.2014.01.011

28. Patel D, Yang YX, Trivedi C, Kavani H, Xie D, Medvedeva E, Lewis J, Khan N. Incidence, Duration, and Management of Anemia: A Nationwide Comparison Between IBD and Non-IBD Populations. Inflamm Bowel Dis. 2020;26(6):934-940.

https://doi.org/10.1093/ibd/izz206

29. Danese S, Hoffman C, Vel S, Greco M, Szabo H, Wilson B, Avedano L. Anaemia from a patient perspective in inflammatory bowel disease: results from the European Federation of Crohn's and Ulcerative Colitis Association's online survey. Eur J Gastroenterol Hepatol. 2014;26(12):1385-91.

https://doi.org/10.1097/MEG.0000000000000200
30. Hou JK, Gasche C, Drazin NZ, Weaver SA, Ehrlich OG, Oberai R, Zapala S, Siegel CA, Melmed G. Assessment of Gaps in Care and the Development of a Care Pathway for Anemia in Patients with Inflammatory Bowel Diseases. Inflamm Bowel Dis. 2017;23(1):35-43. https://doi.org/10.1097/MIB.0000000000000953

31. Qureshi T, Peter Nguyen T, Wang R, Willis D, Shah R, Hou JK. Improving Anemia in Inflammatory Bowel Disease: Impact of the Anemia Care Pathway. Dig Dis Sci. 2019;64(8):2124-2131. https://doi.org/10.1007/s10620-019-05559-w

32. Peyrin-Biroulet L, Lopez A, Cummings JRF, Dignass A, Detlie TE, Danese S. Review article: treating-to-target for inflammatory bowel disease-associated anaemia. Aliment Pharmacol Ther. 2018;48(6):610-617.

https://doi.org/10.1111/apt.14922 